\title{
BMJ Open Patient characteristics, treatment and survival in pulmonary carcinoid tumours: an analysis from the UK National Lung Cancer Audit
}

\author{
Stephanie Hobbins, ${ }^{1}$ Doug West, ${ }^{2}$ Michael Peake, ${ }^{3}$ Paul Beckett, ${ }^{3}$ lan Woolhouse ${ }^{3}$
}

To cite: Hobbins S, West D, Peake M, et al. Patient characteristics, treatment and survival in pulmonary carcinoid tumours: an analysis from the UK National Lung Cancer Audit. BMJ Open 2016;6:e012530. doi:10.1136/bmjopen-2016012530

- Prepublication history for this paper is available online. To view these files please visit the journal online (http://dx.doi.org/10.1136/ bmjopen-2016-012530)

Received 5 May 2016 Revised 30 August 2016 Accepted 1 September 2016

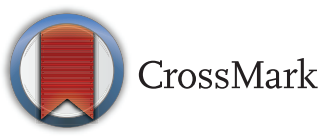

${ }^{1}$ Department of Respiratory Medicine, Queen Elizabeth Hospital Birmingham, Birmingham, UK ${ }^{2}$ Department of Thoracic Surgery, University Hospital Bristol NHS Foundation Trust, Bristol, UK

${ }^{3}$ Care Quality Improvement Department, Royal College of Physicians, London, UK

Correspondence to Dr Stephanie Hobbins; stephaniehobbins@doctors. org.uk

\section{ABSTRACT}

Objectives: Pulmonary carcinoid (PC) is a rare tumour with good prognosis following surgical resection. However, little is known regarding patient characteristics and use of other treatments modalities. Our objective was to review patient characteristics, treatment and survival for patients with PC and contrast these results with other forms of non-small cell lung cancer (NSCLC).

Setting: Audit data from UK National Lung Cancer Audit (NLCA) 2008-2013.

Participants: 184906 lung cancer cases were submitted to the NLCA.

Outcome measures: Primary outcome-survival rates between PC and NSCLC. Secondary outcomedifferences in performance status, lung function and treatment modality between PC and NSCLC.

Results: PC histology was recorded in 1341 (0.73\%) patients and non-carcinoid NSCLC histology in 162959 $(87.4 \%)$ cases. $91 \%$ of patients with PC had good performance status (Eastern Cooperative Oncology Group (ECOG) 0-1), compared with only $53 \%$ of NSCLC. $66 \%$ of PC had localised disease. Of all PC cases, $77 \%$ were treated with surgery, $6.2 \%$ received chemotherapy and $3.6 \%$ received radiotherapy, with the remainder treated with best supportive care. Overall 1-year and 3-year survival rates for $\mathrm{PC}$ were $92 \%$ and $84.7 \%$, respectively. In contrast, 1-year and 3-year survival rates for NSCLC were $36.2 \%$ and $15.6 \%$, However, 3-year survival for PC markedly decreased with worsening performance status and advanced disease to $23.8 \%$ for performance status ECOG 3-4 and 33.6\% for stage IV disease.

Conclusions: In contrast to other forms of NSCLC, the majority of patients with PC present with good performance status, preserved lung function and early stage disease amenable to surgical resection. However, 1 in 5 patients with PC has metastatic disease which is associated with poor prognosis, as is poor performance status at presentation. We believe these data will help clinicians provide accurate prognostic predictions stratified according to patient characteristics at presentation, as well as guide future clinical trials.

\section{INTRODUCTION}

Pulmonary carcinoid (PC) is a rare tumour which is estimated to account for only

\section{Strengths and limitations of this study}

- Very large data set reviewed.

- Highly representative and detailed analysis of pulmonary carcinoid tumours.

- Unable to differentiate between typical and atypical carcinoid tumours.

around $1-2 \%$ of all lung cancers ${ }^{1}$ with an incidence in Europe of 0.2 per 100000 per year. $^{2}$ The pathological definition is usually based on the WHO classification first published in 1967 and most recently updated in the fourth edition. An absence of extensive necrosis and a mitotic count of $<10$ per high power field is used to differentiate PC from high-grade large cell and small cell neuroendocrine tumours. PC can be further subdivided into typical and atypical on the basis of mitotic count and the presence/absence of focal necrosis, although this can only be reliably assessed on a surgical specimen, and there remains interobserver variability in this pathological interpretation. ${ }^{3}$

Overall 5-year survival for lung cancer is poor at $9.5 \%,{ }^{4}$ but PC is a distinct subgroup of tumours which appear to have a more favourable outcome, especially after surgery with a view to a complete R0 resection. ${ }^{2}$ In 2003, a Surveillance, Epidemiology, and End Results (SEER) analysis from the USA reported a 5-year survival of $74 \%$ for patients diagnosed with tracheal and bronchial carcinoid from 1973 to $1999 .{ }^{5}$ A recent SEER analysis of 441 patients with atypical carcinoid reported a surgical resection rate of $78 \%$ with 3-year survival of $67 \% .^{6}$ A smaller case series which included 142 patients with typical and atypical PC reported a surgical resection rate of $72 \%$ with a 5-year survival rate of $89 \%$ for typical carcinoid and $75 \%$ for atypical carcinoid. ${ }^{7}$ Surgical case series tend to report even higher survival rates; a Japanese registry report of tracheal and 
bronchial carcinoid tumours reported a postoperative 10 -year survival of $82 \%$ for typical carcinoid and $59 \%$ for atypical carcinoid. ${ }^{8}$

While these case series provide an interesting insight into the management of carcinoid tumours, the relatively small number of patients and retrospective nature of data collection limit general applicability. In addition, relatively little is known regarding patient characteristics in PC and other treatment modalities such as chemotherapy or radiotherapy. To address this, we performed an analysis of patients with PC submitted to the UK National Lung Cancer Audit (NLCA). We report on patient characteristics, treatments and contrast the results with other forms of non-small cell lung cancer (NSCLC).

\section{METHODS}

Data source

The NLCA is commissioned by the Healthcare Quality Improvement Partnership (HQIP) and collects data on all patients with lung cancer in England and Wales. It was set up to record information about activity, process and outcomes in lung cancer and explain the wide variations in outcome across the UK. ${ }^{9}$ The database includes 112 fields covering patient demographics, referral patterns, investigations, performance status (PS) using the Eastern Cooperative Oncology Group (ECOG) scale, lung function, stage of disease, treatment modalities of surgery (not including endobronchial interventions), chemotherapy and radiotherapy, and survival. Data are collected by an online tool (LUCADA database) from all hospital managing lung cancer in the UK. Annual reports are then sent to all hospital chief executives and medical directors, primary care trusts, cancer commissioners, MDT and network leads. ${ }^{9}$

As this is a review of data from a national audit, no ethical approval is required.

\section{Patient population}

All patients from England and Wales submitted to the NLCA between 2008 and 2013 with recorded histology of PC were included in the analysis. The NLCA database only allows the selection of one Systematized Nomenclature of Human Medicine (SNOMed) III code (M8240/3) for PC which equates to carcinoid 'not otherwise specified'. The data set did not differentiate between typical and atypical carcinoid tumours. All cases were primary pulmonary tumours. No extra-PC tumours or pulmonary metastases from other sites were included.

A comparative analysis was performed using patients diagnosed with non-carcinoid NSCLC (all other cell types excluding small cell cancer).

All staging referred to in this paper is preintervention staging.

\section{Statistical analysis}

Statistical analysis was performed using SPSS V.19. Continuous variables are presented as mean (SD). Survival was calculated using linked office of national statistics data and is presented as proportions at specified time intervals from diagnosis using Kaplan-Meier methods.

\section{RESULTS}

Every hospital in England and Wales participated in the NLCA during the study period and a total of 184906 lung cancer cases were submitted. PC histology was recorded in $1341(0.73 \%)$ patients and NSCLC (as defined in the methods) in $162959(87.4 \%)$ cases.

\section{Patient characteristics}

The patient characteristics for the two groups are shown in table 1.

Age and gender were recorded in all cases. Mean (SD) age at the time of diagnosis for patients with PC was 62 (15) years with $62 \%$ female, whereas mean (SD) age for NSCLC was 72 (11) years, with $44 \%$ female. PS was recorded in $88 \%$ cases and stage in $81 \%$ cases. Ninety-one per cent of patients with PC had good PS (ECOG $0-1$ ) and $66 \%$ had localised disease (stage I-II). In contrast, only $53 \%$ of patients with NSCLC had good PS and 24\% had localised disease. Mean (SD) lung function as measured by the forced expiratory volume in $1 \mathrm{~s}$ $\left(\mathrm{FEV}_{1}\right)$ was $2.27 \mathrm{~L}(0.83)$ in the $\mathrm{PC}$ group and $1.71 \mathrm{~L}$ $(0.72)$ in the NSCLC group.

\section{Treatment}

Treatment data are summarised in table 1. Staging is classified as localised (stage I-II), regional (stage III)

\begin{tabular}{|c|c|c|}
\hline $\mathbf{N}$ & $\begin{array}{l}\text { Carcinoid } \\
1341\end{array}$ & $\begin{array}{l}\text { NSCLC } \\
161618 \\
\end{array}$ \\
\hline \multicolumn{3}{|l|}{ Patient characteristics } \\
\hline Age-mean (SD) & $62(15)$ & $72(11)$ \\
\hline Female & $62 \%$ & $44 \%$ \\
\hline Performance status $0-1$ & $91.4 \%$ & $52.6 \%$ \\
\hline $\mathrm{FEV}_{1}$ 一mean (SD) & $2.27(0.83)$ & $1.71(0.72)$ \\
\hline \multicolumn{3}{|l|}{ Stage } \\
\hline Localised & $65.7 \%$ & $20.4 \%$ \\
\hline Regional & $6.3 \%$ & $22.3 \%$ \\
\hline Metastatic & $8.7 \%$ & $43.1 \%$ \\
\hline Unknown & $19.2 \%$ & $14.2 \%$ \\
\hline \multicolumn{3}{|l|}{ Treatment } \\
\hline Surgery & $76.9 \%$ & $14.2 \%$ \\
\hline Chemotherapy & $6.2 \%$ & $25.7 \%$ \\
\hline Radiotherapy & $3.6 \%$ & $28.4 \%$ \\
\hline No treatment recorded & $13.3 \%$ & $31.7 \%$ \\
\hline
\end{tabular}


Table 2 One-year and 3-year survival rates (\%) stratified according to performance status

\begin{tabular}{llllll}
\hline & \multicolumn{2}{l}{ Carcinoid } & & NSCLC \\
\cline { 2 - 5 } ECOG & 1-year & 3-year & ECOG & 1-year & 3-year \\
\hline $0-1(n=1003)$ & 93.5 & 85.2 & $0-1(n=72065)$ & 55.3 & 26.7 \\
$2(n=70)$ & 75.2 & 57.4 & $2(n=28499)$ & 26.9 & 8.3 \\
3-4 $(n=24)$ & 41.7 & 23.8 & $3-4(n=36528)$ & 11.1 & 2.4 \\
\hline ECOG, Eastern Cooperative Oncology Group; NSCLC, non-small cell lung cancer.
\end{tabular}

and distant (stage IV). Overall, $87 \%$ of the patients with $\mathrm{PC}$ received anticancer treatment (surgery, radiotherapy or chemotherapy). In contrast, $68.3 \%$ of patients with NSCLC received active treatment. The majority of patients with PC $(77 \%)$ were treated with surgery, $6.2 \%$ received chemotherapy and $3.6 \%$ received radiotherapy. This is in contrast to NSCLC, where only $14 \%$ of patients had surgery, $26 \%$ received chemotherapy and a further $28 \%$ received radiotherapy.

\section{Survival}

Overall 1-year and 3-year survival rates for PC were 92\% and $84.7 \%$, respectively. In contrast, 1-year and 3-year survival rates for NSCLC were $36.2 \%$ and $15.6 \%$, respectively. Survival rates by PS are shown in table 2 . There was a stepwise decrease in survival for PC from 1-year and 3-year survival rates of $93.5 \%$ and $85.2 \%$, respectively, for PS 0-1 patients down to $41.7 \%$ and $2.4 \%$, respectively, for poor PS (ECOG 3-4) patients. A very similar pattern of survival rates was seen for localised (stage I-II), regional (stage III) and distant (stage IV) PC disease (table 3). Survival in patients with NSCLC also decreased with worsening PS and more advanced stage, but in contrast to carcinoid, survival rates were much lower (tables 2 and 3).

\section{DISCUSSION}

We analysed data submitted to the NLCA to more clearly define patient characteristics, treatment modalities and survival of this subgroup of lung cancers, which we contrasted to patients with non-carcinoid NSCLC. We confirmed that PC is a rare tumour and constituted $<1 \%$ of all lung cancers in our data set. We demonstrated that the majority $(68 \%)$ of patients with $\mathrm{PC}$ are female, which is in keeping with the previous SEER database reports, ${ }^{56}$ but higher than smaller case series ${ }^{10}{ }^{11}$ which likely reflects the more representative nature of the
NLCA and SEER databases. We report, for the first time, that patients with PC have excellent PS at presentation and well-preserved lung function. In addition, the majority $(66 \%)$ of patients with $\mathrm{PC}$ in this study presented with early stage (localised) resectable disease, although $10 \%$ had metastatic disease at presentation. These findings are largely consistent with the stage distribution reported in the SEER analysis of tracheal and bronchial $\mathrm{PC}^{5}$ where two-thirds of patients had localised disease at presentation; however, the proportion of patients with metastatic disease in this study was only $0.5 \%$ for the period 1992-1999. This could be explained by selective submission of surgical cases, whereas data submitted to the NLCA are for all patients with lung cancer, irrespective of treatment modality. The patient characteristics for the PC group were in stark contrast to the NSCLC group. The mean age for the patients with NSCLC was 10 years older than the PC group and only half of patients had good PS. The lung function, as measured by $\mathrm{FEV}_{1}$, was $25 \%$ lower than the PC group. Similarly, only one in four patients in the NSCLC group presented with localised disease and over $40 \%$ had metastatic disease at presentation.

Surgery is recommended as first-line treatment in locoregional PC. ${ }^{3}{ }^{12}$ In our study, three-quarters of patients with PC underwent surgery, reflecting the stage distribution and fitness of this group of patients. The treatment of metastatic PC is less clearly defined. Systemic therapy is generally recommended for metastatic disease; however, the results with chemotherapy have been largely disappointing. ${ }^{3}$ Given that chemotherapy is not routinely used or recommended in the adjuvant setting as it does not improve survival, ${ }^{13}$ we can infer from our results that most patients with metastatic disease are treated with chemotherapy, despite the poor reported response rates with this treatment. To the best of our knowledge, this is the first time that the use of chemotherapy has been reported on a large scale for

Table 3 One-year and 3-year survival rates (\%) stratified according to stage

\begin{tabular}{llllll}
\hline & \multicolumn{2}{l}{ Carcinoid } & & \multicolumn{2}{c}{ NSCLC } \\
\cline { 2 - 5 } STAGE & 1-year & 3-year & STAGE & 1-year & 3-year \\
\hline Localised $(n=429)$ & 96.4 & 87.7 & 63.1 & Localised $(n=33040)$ & 73.0 \\
Regional $(n=85)$ & 75.8 & 33.6 & Regional $(n=26$ 070) & 40.9 & 12.4 \\
Metastatic $(n=117)$ & 54.9 & Metastatic $(n=69598)$ & 16.7 & 3.5 \\
\hline ECOG, Eastern Cooperative Oncology Group; NSCLC, non-small cell lung cancer.
\end{tabular}


patient with PC tumours. Similarly, there is very little in the literature relating to radiotherapy treatment in PC and recent guidelines do not refer to this treatment modality. ${ }^{3}$ The recent report by Steuer and colleagues found that $12.5 \%$ of patients with atypical PC received radiotherapy which is higher than the $3.6 \%$ radiotherapy treatment rate found in our study and is likely due to the more aggressive nature of atypical PC tumours.

Our data confirm that the prognosis for PC is generally excellent, with overall 1-year and 3-year survival rates of $92 \%$ and $84.7 \%$, respectively. Once again, this is in stark contrast to other forms of NSCLC where the 1-year survival rate was only $36.2 \%$. We found that stage at presentation in PC is an important determinant of survival which is in keeping with the findings of previous studies, ${ }^{5}{ }^{6}$ but we also report that PS is an equally important predictor of survival for patients with PC. This complements recent work from the European Association of Thoracic Surgeons (ESTS) who found PS was a predictor for mortality in this group. ${ }^{14}$ Although the majority of our patients with PC have good PS at presentation, the small proportion with poor PS in our study had a 1-year survival of only $42 \%$. This group of patients may have had other comorbidities rendering them unfit for any form of active anticancer treatment which could explain the poor survival.

The current study has a number of strengths but also potential limitations. The data set provided by the NLCA is large, with mostly excellent levels of data completeness, but there are some missing data; and also no data were available on patients presenting with carcinoid syndrome. Additionally no information was available on regional control rates or treatment failure. Although the missing data could impact on results, we feel this is likely offset by the size of the data pool. A further limitation is the inability to discriminate between typical and atypical carcinoid tumours in this data set.

However, it is felt that overall it provides a highly representative and detailed analysis of all forms of PC tumours, irrespective of treatment given. However, the inability to record typical versus atypical carcinoid histology limits the study's ability to differentiate treatment and outcomes by these two cell types. Also, the data available categorise cancer by stage rather than T, N, M status. In addition, the details of chemotherapeutic regimens and radiotherapy dosing schedules were not recorded in the NLCA during the time of this analysis.

In conclusion, our large and detailed data set demonstrate that, in contrast to other forms of NSCLC, the majority of patients with PC present with good PS, well-preserved lung function and early stage disease leading to high rates of surgical resection. However, one in five patients have local or regional metastatic disease which is associated with a poor prognosis, as is poor PS at presentation. We believe that these data will help clinicians provide more accurate prognostic predictions stratified according to patient characteristics at presentation. The data shown here provide reassurance to patients and clinicians that patients with carcinoid with early disease who have surgery enjoy high levels of survival; however, clinical trials are needed of newer treatment in metastatic disease.

Acknowledgements Mr Arthur Yelland, Senior Information Analyst, Health and Social Care Information Centre.

Contributors All authors provided substantial contributions to the work. IW, MP, PB devised the study and supervised analysis/write up. DW provided surgical input into analysis and interpretation of the results. SH analysed results and wrote up study findings. All authors were involved in critical revision of draft manuscripts and all were happy with final version and approved it for submission for publication. All authors are happy to be accountable for all aspects of the work submitted.

Funding The NLCA is funded by the Health Quality Improvement Partnership (HQIP).

Competing interests None declared.

Provenance and peer review Not commissioned; externally peer reviewed.

Data sharing statement No additional data are available.

Open Access This is an Open Access article distributed in accordance with the Creative Commons Attribution Non Commercial (CC BY-NC 4.0) license, which permits others to distribute, remix, adapt, build upon this work noncommercially, and license their derivative works on different terms, provided the original work is properly cited and the use is non-commercial. See: http:// creativecommons.org/licenses/by-nc/4.0/

\section{REFERENCES}

1. Öberg $\mathrm{K}$, Hellman $\mathrm{P}$, Ferolla $\mathrm{P}$, et al, on behalf of the ESMO Guidelines Working Group. Neuroendocrine bronchial and thymic tumors: ESMO Clinical Practice Guidelines for diagnosis, treatmen and follow-up. Ann Oncol 2012;23(Suppl 7):vii120-3.

2. Morandi U, Casali C, Rossi G. Bronchial typical carcinoid tumors. Semin Thorac Cardiovasc Surg 2006;18:191-8.

3. Caplin ME, Baudin E, Ferolla P, et al, ENETS consensus conference participants. Pulmonary neuroendocrine (carcinoid) tumors: European Neuroendocrine Tumor Society expert consensus and recommendations for best practice for typical and atypical pulmonary carcinoids. Ann Oncol 2015;26:1604-20.

4. http://www.cancerresearchuk.org/cancer-info/cancerstats/types/lung/ survival/lung-cancer-survival-statistics

5. Modlin IM, Lye KD, Kidd M. A 5-decade analysis of 13,715 carcinoid tumors. Cancer 2003;97:934-59.

6. Steuer CE, Behera M, Kim S, et al. Atypical carcinoid tumor of the lung: a surveillance, epidemiology, and end results database analysis. J Thorac Oncol 2015;10:479-85.

7. Fink G, Krelbaum T, Yellin A, et al. Pulmonary carcinoid: presentation, diagnosis, and outcome in 142 cases in Israel and review of 640 cases from the literature. Chest 2001;119:1647-51.

8. Soga J, Yakuwa Y. Bronchopulmonary carcinoids: an analysis of 1,875 reported cases with special reference to a comparison between typical carcinoids and atypical varieties. Ann Thorac Cardiovasc Surg 1999;5:211-19.

9. Beckett $\mathrm{P}$, Woolhouse I, Stanley $\mathrm{R}$, et al. Exploring variations in lung cancer care across the UK-the 'story so far' for The National Lung Cancer Audit. Clin Med (Lond) 2012;12:14-18.

10. Cooper WA, Thourani VH, Gal AA, et al. The surgical spectrum of pulmonary neuroendocrine neoplasms. Chest 2001;119:14-18.

11. Hurt R, Bates M. Carcinoid tumours of the bronchus: a 33 year experience. Thorax 1984;39:617-23.

12. Phan AT, Oberg K, Choi J, et al. NANETS consensus guideline for the diagnosis and management of neuroendocrine tumors: well-differentiated neuroendocrine tumors of the thorax (includes lung and thymus). Pancreas 2010;39:784-98.

13. Nussbaum DP, Speicher PJ, Gulack BC, et al. Defining the role of adjuvant chemotherapy after lobectomy for typical bronchopulmonary carcinoid tumors. Ann Thorac Surg 2015;99:428-34.

14. Filosso P, Guerrera F, Evangelista A, et al. Prognostic model of survival for typical bronchial carcinoid tumours: analysis of 1109 patients on behalf of the European Association of Thoracic Surgeons (ESTS) Neuroendocrine Tumours Working Group. Eur J Cardiothorac Surg 2015;48:441-7. 